\section{EGFR's new tricks}

\author{
By Lev Osherovich, Senior Writer
}

A pair of cell culture studies suggests that epidermal growth factor receptor promotes cancer through mechanisms beyond kinase activity. According to company representatives and researchers contacted by SciBX, the findings point to new ways of boosting the efficacy of approved drugs that target epidermal growth factor receptor-small molecule tyrosine kinase inhibitors and mAbs. However, the results must be validated with further studies in human tumors.

Epidermal growth factor receptor (EGFR) is a transmembrane receptor with an intracellular tyrosine kinase domain. Normally activated by growth factors, EGFR molecules dimerize and phosphorylate one another, turning on signaling pathways that drive cell division. However, excessive EGFR signaling can lead to many cancers, including non-small cell lung cancer (NSCLC), colorectal cancer and glioblastoma multiforme (GBM).

One of the two studies, published in Cancer Cell by University of Texas M.D. Anderson Cancer Center researchers, shows that EGFR is a partner to a cancer-associated sugar transporter. ${ }^{1}$ The other study, published in Nature Cell Biology by McGill University researchers, shows that a tumor-associated mutant form of EGFR can spread from cell to cell via membranous vesicles called oncosomes. ${ }^{2}$

\section{Sugar daddy}

The results reported in Cancer Cell by the research groups of Mien-Chie Hung, professor of molecular and cellular oncology, and Isaiah Fidler, professor of cancer biology, may explain why inhibiting EGFR kinase activity is not enough to kill most tumors.

In cultured human prostate cancer cells, the team found that EGFR binds to and stabilizes a glucose-pumping protein called sodium/glucose cotransporter 1 (SGLT1) on the cell surface. Cancer cells are especially dependent on glucose for energy, and SGLT1 is a major route of glucose absorption in tumors. ${ }^{3}$

The interaction between EGFR and SGLT1 helps cancer cells gorge on glucose. High EGFR expression correlated with high SGLT1 levels and allowed tumors to survive in low glucose conditions via more efficient uptake of sugar by the transporter.

When EGFR was knocked down by short interfering RNA, SGLT1 levels fell, leading to death by autophagy, a process of self-digestion triggered by cellular starvation. This drop in SGLT1 was likely due to proteasomal degradation, the researchers found.

Different parts of EGFR were required for binding to SGLT1 and for sending cancer-associated proliferative signals. Whereas EGFR's kinase activity resided in the protein's intracellar domain, the extracellular domain bound SGLT1.
"EGFR is primarily a kinase, but we found that this interaction with SGLT1 is independent of the kinase activity," said Hung.

To prove that kinase activity is not needed for SGLT1 stabilization, the team engineered a mutant form of EGFR with an enzymatically dead kinase domain. Cells with kinase-compromised EGFR stopped growing but still survived. In contrast, entirely eliminating EGFR using siRNA led to autophagic cell death, paralleling what happened when SGLT1 was knocked down.

\section{Same target, different mechanisms}

The Cancer Cell findings could shed light on how the two classes of approved EGFR inhibitors-small molecules and antibodies-may work differently.

The approved small molecule EGFR inhibitors, Iressa gefitinib from AstraZeneca plc and Tarceva erlotinib from OSI Pharmaceuticals Inc., Genentech Inc. and Roche, are primarily effective in patients who have mutations in the EGFR kinase domain that result in constitutive activation of the receptor. However, most patients with high levels of nonmutated EGFR do not respond to the tyrosine kinase inhibitors. ${ }^{4}$

Whereas small molecules block the mitogenic signaling activities of EGFR, mAbs block the extracellular surface of the receptor, potentially preventing interactions with SGLT1.

Rafael Amado, executive director of global development at Amgen Inc., said the Cancer Cell paper "explains some of the differences in kinase inhibitors versus $\mathrm{mAb}$ therapeutics." He suggested that some tumors could be highly dependent on SGLT1 and would thus be more susceptible to disruption by anti-EGFR mAbs.

"There were already some clues that EGFR activity was independent" of the receptor's kinase domain, said Amado. "This solidifies that concept."

Amgen's Vectibix panitumumab is approved to treat colorectal cancer.

"I think it's a very significant finding," said Eric Rowinsky, CMO and EVP of ImClone Systems Inc. "This could be a substantial lead in understanding how best to target EGFR."

ImClone's Erbitux cetuximab is approved to treat colon cancer and head and neck cancer. The mAb is partnered with Bristol-Myers Squibb Co. and Merck KGaA.

Indeed, Hung told $S c i B X$ that when his team used $\mathrm{mAb}$ therapeutics in their assay, "the outcome is quite different" from assays performed with small molecules.

Several small molecule kinase inhibitors could not kill tumor cells despite inhibiting kinase activity. Although the activity of mAbs was not discussed in the paper, Hung mentioned to SciBX unpublished data that showed blocking EGFR's extracellular domain with an $\mathrm{mAb}$ reduced the receptor's interaction with SGLT1 and killed the tumor cells. He suggested this may be because the mAbs blocked the interaction between EGFR and SGLT1.

According to OSI scientists, the study raises interesting ideas about how tumors may survive low-glucose conditions, but the company thinks further experiments on the role of glucose transport in EGFR-driven tumors are required. 
Moreover, the company thinks the finding that kinase inhibitors do not kill cancer cells could be a benefit. In an e-mail to SciBX, the researchers said Tarceva acts as a cytostatic agent specific for tumor cells instead of broadly killing both cancerous and healthy cells like conventional chemotherapeutics.

\section{Apply here}

Amgen's Amado suggested that, at least initially, SGLT1 could be used as a diagnostic marker. SGLT1 has previously been found in certain tumors, ${ }^{5}$ but its importance has not been recognized until now.

"I don't know whether colorectal tumors are more dependent on SGLT1, but there will probably be a rush to identify susceptible tumors" that overexpress this protein, Amado said. Sorting out whether SGLT1 levels correlate with drug responsiveness will come next, he added.

Beyond diagnostics, a therapeutic tactic would be to directly target SGLT1 either alone or in combination with approved EGFR drugs. Before doing that, Hung wants to test the effect of inhibiting SGLT1's glucose transport activity in an animal model of cancer.

Glucose transport is the target of at least one therapeutic candidate: 2DG from Threshold Pharmaceuticals Inc. The compound is a mimic of glucose that cannot be metabolized and competes with glucose for transport into cancer cells. 2DG is in Phase I trials to treat solid tumors. However, numerous studies indicate 2DG may target other glucose transporters besides SGLT1.

ImClone's Rowinsky suggested that combining $\mathrm{mAb}$ therapeutics with glucose transport inhibitors or competitors could be done as a proof-ofprinciple study in patients where other therapeutics had failed.

"The real proof would be a randomized trial," said Rowinsky. "I cannot imagine that one wouldn't step in that direction."

The findings reported in the Cancer Cell paper are not patented, according to Hung. However, he is "interested in working with industry" to develop SGLT1-blocking molecules.

\section{Transformation transfer}

Meanwhile, a Nature Cell Biology paper suggests that a mutant form of EGFR associated with GBM can spread from cell to cell. The study shows that an infusion of EGFR protein delivered by secreted extracellular microvesicles called oncosomes promotes transformation in quiescent tumor cells.

Oncosomes may be a new vector for spreading cancer within the body, according to the study's lead author, Janusz Rak, associate professor of pediatrics at McGill.

"Once cells become transformed, they trigger a vesiculation process in which the cancer proteins spread" through secreted oncosomes, Rak said. The structures seem to be active, triggering downstream events like signaling pathway activation, he added.

Rak's team discovered oncosomes while studying how transfection with EGFR variant III (EGFRvIII), a truncated and hyperactive version of EGFR found in many GBM tumors, affected the appearance and growth of cultured human glioma cells.

Compared with what was seen in mock-treated controls, cells transfected with EGFRvIII triggered the secretion of oncosomes, which consist of lipids and membrane-associated proteins. The structures were visible in electron micrographs and could be detected in the cell culture medium of EGFRvIII-transfected cells, allowing Rak's team to test the effect of oncosomes on untransformed cells.

The team purified oncosomes and tested their effect on nondividing glioma cells. When the oncosome preparation was added to the culture medium, the cells absorbed them and turned on growth genes known to be regulated by EGFR. Unlike mock-treated controls, cells exposed to oncosomes proliferated aggressively in a cell culture assay of metastasis.

The researchers also found that mice injected with EGFRvIII-bearing glioma cells developed aggressive tumors and had EGFRvIII-bearing oncosomes in their blood. In contrast, mice injected with mock-treated glioma cells did not develop tumors.

Evidence that transformation can be transmitted in this fashion in humans has not yet emerged. However, the study could explain why EGFRvIII-driven tumors are so aggressive in the clinic, according to Rak.

"Usually, only a minority of cells express EGFRvIII, but it has an impact on the entire tumor," Rak told SciBX.

According to Rak, this may be because the majority of cells are passive followers, programmed to proliferate by oncosomes secreted by a minority of "ringleader" cells.

John Bell, professor of medicine and biochemistry at the University of Ottawa and CSO of Jennerex Inc., offered a similar hypothesis.

"If oncoprotein spreading is going on, it means that you need to target the tumor-initiating cells" and not the surrounding cells, he said. "The target subpopulation of EGFRvIII-producing cells could be really small."

Jennerex targets EGFR mutant tumor cells with JX-963, an oncolytic virus adapted from vaccinia that has an affinity for cells that are proliferating due to excessive EGFR signaling. Once inside tumor cells, JX-963 enhances the immune response against them. The agent is in preclinical development to treat lung, renal and prostate cancers.

Venky Ramakrishna, assistant director of immunology at Avant Immunotherapies Inc. called the Rak study "a novel piece of work" that builds on earlier discoveries of oncosome-like structures in the immune system.

Avant's CDX-110 is a peptide vaccine that directs immune cells toward cells expressing EGFRvIII. The vaccine is in Phase II trials for GBM and is partnered with Pfizer Inc. Avant acquired CDX-110 as a result of its merger with Celldex Therapeutics Inc. on March 17.

Ramakrishna suggested that oncosomes could be an abnormal version of an intercellular communication mechanism used by the immune system. He said the transfer of proteins by Rak's oncosomes resembles antigen transfer in the lymph nodes. ${ }^{6}$

In antigen transfer, dendritic cells gather antigens in the periphery and then pass microvesicles containing these foreign proteins to lymphocytes, eliciting an immune response.

Ramakrishna told SciBX that Rak's findings need to be validated in human tissues and more sophisticated animal models before oncosomes can be considered as therapeutic targets. He also recommended searching for other tumor-specific proteins associated with oncosomes. 
Although the demonstration of cancer transfer in cell culture is promising, "it's another thing to find it in human cancers," he said.

To address these concerns, Rak's team is developing improved diagnostic methods with the aim of detecting oncosomes in the blood of GBM patients.

Rak also wants to identify other membrane-bound proteins needed for the secretion and spread of oncosomes.

Rak has filed for patents on diagnostic techniques based on this work through McGill University's Office of Technology Transfer.

\section{REFERENCES}

1. Weihua, Z. et al. Cancer Cell; published online May 4, 2008; doi:10.1016/j.ccr.2008.03.015

Contact: Isaiah J. Fidler, University of Texas M.D. Anderson Cancer Center, Houston, Texas

e-mail: ifidler@mdanderson.org

Contact: Mien-Chie Hung, same affiliation as above e-mail: mhung@mdanderson.org

2. Al-Nedawi, K. et al. Nat. Cell Biol.; published online April 20, 2008; doi:10.1038/ncb1725
Contact: Janusz Rak, McGill University, Montreal, Quebec, Canada e-mail: janusz.rak@mcgill.ca

3. Ishikawa, N. et al. Jpn. J. Cancer Res. 92, 874-879 (2001)

4. Sharma, S.V. et al. Nat. Rev. Cancer 7, 169-181 (2007)

5. Helmke, B.M. et al. Oral Oncol. 40, 28-35 (2004)

6. Li, X.B. et al. J. Cell. Mol. Med. 10, 364-375 (2006)

COMPANIES AND INSTITUTIONS MENTIONED

Amgen Inc. (NASDAQ:AMGN), Thousand Oaks, Calif. AstraZeneca plc (LSE:AZN; NYSE:AZN), London, U.K.

Avant Immunotherapies Inc. (NASDAQ:AVAN), Needham, Mass.

Bristol-Myers Squibb Co. (NYSE:BMY), New York, N.Y.

Genentech Inc. (NYSE:DNA), South San Francisco, Calif. ImClone Systems Inc. (NASDAQ:IMCL), New York, N.Y.

Jennerex Inc., San Francisco, Calif.

Merck KGaA (XETRA:MRK), Darmstadt, Germany

McGill University, Montreal, Quebec, Canada

OSI Pharmaceuticals Inc. (NASDAQ:OSIP), Melville, N.Y.

Pfizer Inc. (NYSE:PFE), New York, N.Y.

Roche (SWX:ROG), Basel, Switzerland

Threshold Pharmaceuticals Inc. (NASDAQ:THLD), Redwood City, Calif.

University of Ottawa, Toronto, Ottawa, Canada

University of Texas M.D. Anderson Cancer Center, Houston, Texas 